\title{
Quantification of Artifacts in MR Images Caused by Commonly Used
}

\section{Dental Materials}

\author{
Solmaz Valizadeh, ${ }^{1}$ Hamidreza Pouraliakbar, ${ }^{2}$ Vida Arzani, ${ }^{1,{ }^{*}}$ and Ali Nahardani ${ }^{3}$ \\ ${ }^{1}$ Department of Radiology, School of Dentistry, Shahid Beheshti University of Medical Sciences, Tehran, Iran \\ ${ }^{2}$ Rajaie Cardiovascular Medical and Research Center, Iran University of Medical Sciences, Tehran, Iran \\ ${ }^{3}$ School of Allied Medical Sciences, Shahid Beheshti University of Medical Sciences, Tehran, Iran \\ "Corresponding author: Vida Arzani, Department of Radiology, School of Dentistry, Shahid Beheshti University of Medical Sciences, Tehran, Iran. Tel: +98-2122439982, Fax: \\ +98-2122439784, E-mail: vidaarzani@sbmu.ac.ir
}

Received 2016 December 16; Revised 2017 February 09; Accepted 2017 March 09.

\begin{abstract}
Background: Although new approaches for application of magnetic resonance imaging (MRI) in dentistry have been promising, the presence of dental materials in the oral cavity represents a major obstacle to generating quality MRI data. The resulting artifacts can adversely affect diagnostic accuracy of oral and maxillofacial conditions.

Objectives: This study aimed to detect and assess artifacts caused by metallic and non-metallic dental materials in MR images. Materials and Methods: Nineteen commonly used dental materials were used in an in vitro assay. An empty cube-shaped phantom with internal dimensions of $15 \times 15 \times 15 \mathrm{~cm}^{3}$ was fabricated of polyester and filled with paraffin or distilled water to create optimal contrast for T1-weighted (T1W; for anatomical structures), T2-weighted (T2W, for pathological conditions) and proton densityweighted (PDW) MR images. Dental material samples were manufactured and placed at the center of the mold using polyester tubes. All samples were then subjected to MRI using two-dimensional (2D) and three-dimensional (3D) T1W, T2W and PDW imaging sequences. The data were subjected to one-way analysis of variance (ANOVA) followed by mean comparison using the Tukey's test. Results: Comparison of mean artifact size in 2D and 3D images showed that ceramic caused the smallest $\left(189.48 \mathrm{~mm}^{3}\right)$ and orthodontic metal brackets caused the largest $\left(4307.5 \mathrm{~mm}^{3}\right)$ artifact areas. Assessment of the amount of artifacts created in different imaging sequences revealed minimal artifact in inversion recovery sequence and maximum artifact in gradient echo sequence of the amount of artifacts at different weighing times in 2D and 3D images showed minimal artifacts in PDW and maximum artifacts in T2W.

Conclusion: Bracket, titanium implants, and the nickel-chromium ( $\mathrm{Ni}-\mathrm{Cr}$ ) materials are the three most susceptible materials to external Bo magnetic field; thus creating huge metal-induced artifacts. The Siemens default two dimensional turbo spin echo (2D-TSE), 2D-BLADE, three dimensional volumetric interpolated breath-hold examination (3D-VIBE) and three dimensional sampling perfection with application optimized contrasts using different flip angle evolution (3D-SPACE) are the most appropriate pulse sequences for the 2D-T1W, 2D-T2W, 3D-T1W, and 3D-T2W evaluations of the bracket/titanium / Ni-Cr materials, respectively. The rest of the materials' magnetic susceptibility effects are negligible, and artifacts are small.
\end{abstract}

Keywords: Magnetic Resonance Imaging, Dental Materials, Artifact

\section{Background}

Magnetic resonance imaging is a modality that provides detailed images of tissues and organs without using X-ray or ionizing beams. These advantages as well as higher contrast than other X-ray-based imaging systems such as computed tomography (CT), have resulted in extensive use of MRI (1). At present, MRI is also used in dentistry. The main dental applications of MRI include evaluation of extra-cranial tumors, assessment of morphology and function of temporomandibular joint, implant treatment planning, evaluation of dentomaxillofacial anatomy and pathological lesions in this region, particularly in jaws bone marrow spaces, evaluation of impacted teeth and trigeminal and mandibular nerve canals, treatment planning for orthodontic and orthognathic surgeries, detection of dental caries and assessment of dental pulp (2).

Despite numerous advantages of MRI, studies on its dental applications are limited due to limited access of dentists to MRI and artifacts caused by dental materials (2). The major source of maxillofacial MR imaging artifacts is the magnetic susceptibility effect of different local materials which is mainly depicted by a regional signal loss. The magnetic susceptibility effect is defined as a material's potential to become magnetized in a magnetic environment, under the influence of the source magnetic field strength. Every material becomes magnetized in a large magnetic field, on this basis the materials are categorized 
into three major classes, i.e. diamagnetic, paramagnetic and ferromagnetic. Diamagnetic materials have little effects on the MRimages quality, but the paramagnetic materials can induce heavy image distortions and signal loss in their local environments. Human body tissues are mainly diamagnetic, whereas the magnetic susceptibility effects of dental materials are unknown due to different components of the alloys (3). The flow void signal around dental materials could be mistaken as air in $\mu$-map (4). Based on the introduction of new fast imaging techniques (e.g. T1/T2 weighted turbo spin echo (TSE) and T1-weighted 3D fast low angle shot magnetic resonance imaging (3D FLASH MRI pulse sequences) and new formulations of dental materials (thus their unknown effect on image quality) motivated the scientists to conduct more extensive researches in maxillofacial MRI (2).

In the recent years, limited studies have evaluated artifacts caused by dental materials. Klinke et al, (5) in 2012 evaluated the artifacts caused by mainly metallic dental materials on MR images and CT scans. They noticed that metallic restorative materials had a more significant effect on CT scans than on MR images and rare earth elements used in composites created artifacts on both CT and MR images.

In 2013, Timofiyeva et al. (6) evaluated the effect of dental materials on MR images in vitro and in vivo and showed that dental materials could be classified into three groups of compatible, I compatible and incompatible in this respect. Incompatible dental materials should not be present in the oral cavity during MRI.

The current literature has generally focused on artifacts caused by metallic dental materials in the oral cavity, but a holistic picture of the effects of non-metallic dental materials on MR images in terms of creating artifacts is not yet available.

\section{Objectives}

Thus, this study aimed to detect and assess artifacts created by different metallic and non-metallic dental materials on MR images and to evaluate the effects of different MRI parameters on artifact size.

The data would enable radiologists and clinicians to quantify loss of diagnostic accuracy due to artifacts created by dental materials in the imaging modality and take them into account for clinical decision making. In addition, such information would help manufacturers in production of dental materials causing fewer artifacts on MR images.

\section{Materials and Methods}

An in vitro study was conducted on 19 commonly used dental materials in different fields of dentistry, and one sample of each dental material was fabricated to undergo MRI. Imaging was done in the imaging department of Shahid Rajaee educational and research center using MAGNETOM Avanto 1.5T MRI system (Siemens Medical Solutions, Erlangen, Germany); MR images were analyzed using Syngo MR B15 software (Siemens Medical Solutions, Erlangen, Germany) and MATLAB (MATLAB and Image Processing Toolbox Release 2015, The MathWorks, Inc., Natick, Massachusetts, United States) software programs.

A total of 19 samples were fabricated of commonly used dental materials (metallic and non-metallic) as follows:

1) Amalgam (SDI, Victoria, Australia)

2) Composite resin (point4; Kerr, Orange, CA and Esthet-X; Dentsply/Caulk, Milford, DE and Vitalescence; Ultradent, South Jordan, UT, USA)

3) Composite resin (Z100; 3M ESPE, Seefeld, Germany)

4) Glass Ionomer (Fuji II LC; GC International Corp., Tokyo, Japan)

5) Glass Ionomer (ChemFil Superior; Dentsply De Trey, Konstanz, Germany)

6) Zinc oxide eugenol (ZOE) paste (Zonalin; Kemdent, Purton, Swindon, Wiltshire, UK)

7) Endodontic sealer (AH 26; Dentsply De Trey GmbH, Konstanz, Germany)

8) Gutta-Percha (Ariadent, Tehran, Iran)

9) Gutta-Percha (Gapadent, JIAFA.CO; LTD, South Korea)

10) Temporary cement (Temp Bond/Temp Bond NE; Kerr; Orange, CA, USA)

11) Cavit (Coltosol; Ariadent, Tehran, Iran)

12) Calcium hydroxide (Dycal; Dentsply Caulk, Milford, DE, USA)

13) Mineral trioxide aggregate (MTA) (Dentsply, Tulsa Dental, Tulsa, OK, USA)

14) Zinc-phosphate cement (Harvard Cement; Richer and Hoffmann Harvard-Dental, Gesellschaft, Berlin, Germany)

15) Polycarboxylate cement (Harvard Dental International, GmbH, Germany)

16) IPS e.max Press (lithium disilicate glass-ceramic LT ingot; Ivoclar-Vivadent, Zurich, Switzerland)

17) Nickel-chromium (Ni-Cr) dental alloy (Unitech, savigny/orge, France)

18) Orthodontic metal bracket (Shark MBT 022; World Class Orthodontics, Lindenberg/Allgau, Germany)

19) Titanium implant fixture (Biomet 3i, FL, USA)

Samples were fabricated in cylindrical form with 0.5 $\mathrm{cm}$ diameter and length. For this purpose, a cylindrical mold with an internal diameter of $5 \mathrm{~mm}$ and height 
of $5 \mathrm{~mm}$ was used. An empty cubic phantom with internal dimensions of $15 \times 15 \times 15 \mathrm{~cm}^{3}$ was fabricated of polyester. To obtain favorable contrast for T1w, T2w and proton density-weighted (PDW) imaging, the phantom was filled with paraffin or distilled water and dental materials were placed at the center of the phantom using polyester tubes (Figure 1A).

First, the samples were precisely inspected by two observers, an oral and maxillofacial radiologist and a MRI physicist, to ensure that the samples were sound. Imaging risks and compatibility of the samples were then analyzed to ensure no damage to the research environment/equipment in a way that samples were tested by a 0.1 Tesla magnet to ensure that they are not adhere to magnetic field. The samples were then placed on the MRI examination table. After connecting the 8-channel phased array coil for brain imaging and the pads, the sample was slid into the center of the magnet and initial scanning was commenced. First, a pilot sample was scanned to assess artifacts caused by the polyester cylinder. Due to absolute compatibility of the cylinder, the control sample was excluded. Then, all samples were scanned using 2D and 3D T1W (used for anatomical structures), T2W (used for pathological conditions) and PDW imaging sequences according to Table 1 . T1W sequences included two dimensional spin echo (2D-SE), two dimensional turbo spin echo (2D-TSE), two dimensional fast low angle shot (2D-FLASH), 3D-FLASH and three dimensional volumetric interpolated breathhold examination (3D-VIBE). T2W sequences consisted of 2D-TSE, two dimensional true fast imaging with steadystate precession (2D-True FISP) two dimensional fluid attenuated inversion recovery (2D-FLAIR), 2D-BLADE, 3D-TSE and three dimensional sampling perfection with application optimized contrasts using different flip angle evolution (3D-SPACE). The PDW sequence used in this study was 2D-TSE. Each sample was scanned in three orthogonal directions ( $\mathrm{X}, \mathrm{Y}$ and $\mathrm{Z}$ ) by changing the phase and frequency encoding gradients orientations. They were considered as the three replications to conduct statistical analysis and their means were used for mean comparison.

All the MR images were controlled acquired under the supervision of an oral and maxillofacial radiologist in the aspects of spatial resolution, contrast resolution and absence of undesirable imaging artifacts (e.g. aliasing artifact, image distortion, cross contamination etc.) (Figure $1 \mathrm{~B}$ and $\mathrm{C}$ ). Additional MR images of different materials are presented in Supplementary File Appendix 1 and 2. After ensuring optimal quality of images, they were transferred to MATLAB software and the size of artifacts was quantified by a MR clinical physicist. Images were converted to binary (black and white) images using Otsu algorithm and artifactual points were visualized as absolutely black points (known as segmentation/binarization). Next, number of all artifactual pixels in three mutually perpendicular Cartesian axes (x, y, z perpendicular axes) was counted and based on their dimensions; volumetric dimensions of magnetic susceptibility artifacts (blooming) were calculated by the multiplication of the number of absolutely black voxels into the dimension of each voxel. The resultant quantity dimension was in cubic millimeters.

The data were analyzed using SPSS version 21 (SPSS Inc., IL, USA). The mean and standard deviation of the size of artifacts for each material in different imaging conditions were calculated, and the groups were compared in terms of the mean size of artifacts. Tukey's post-hoc test was applied for pairwise comparison of the groups at $\mathrm{P}=0.05$ level of significance.

\section{Results}

Comparison of the mean size of artifacts of dental materials on 2D images showed that ceramic and orthodontic metal brackets caused the smallest and largest artifact volumes, respectively. The polycarboxylate cement and orthodontic metal brackets showed the smallest and the largest artifactual volumes in the final 3D data analysis orderly. Although, the 2D and 3D overall analysis showed the same results, the artifact size of polycarboxylate cement was relatively smaller than ceramic (Table 2, Figure 2).

Comparison of the mean size of artifacts on T1w images showed that ceramic and orthodontic metal brackets created the smallest and largest artifact areas, respectively. Comparison of the mean size of dental artifacts on T2w images showed that Fuji II glass ionomer, ceramic, polycarboxylate and Z100 composite created the smallest and orthodontic metal brackets caused the largest artifact areas. Comparison of the mean size of artifacts on PDw images revealed that Fuji II glass ionomer created the smallest and orthodontic metal brackets caused the largest artifact areas (Figure 3).

Optimal conditions and parameters of imaging to minimize artifacts caused by each dental material are presented in Table 3.

The difference in the size of artifacts based on imaging sequences was analyzed using ANOVA. To eliminate calculation errors, $\mathrm{Ni}-\mathrm{Cr}$, implant and bracket were excluded from calculations since they created large artifacts and were separately analyzed because they could cause a bias in the final results. For dental materials causing insignificant artifacts, 2D T1w sequences showed a significant difference in size of artifacts $(\mathrm{P}<0.0001)$ such that the FLASH sequence resulted in the smallest artifact possible. A significant difference was also found in artifact size in 3D T1w images of dental materials with insignificant artifacts $(\mathrm{P}=$ 


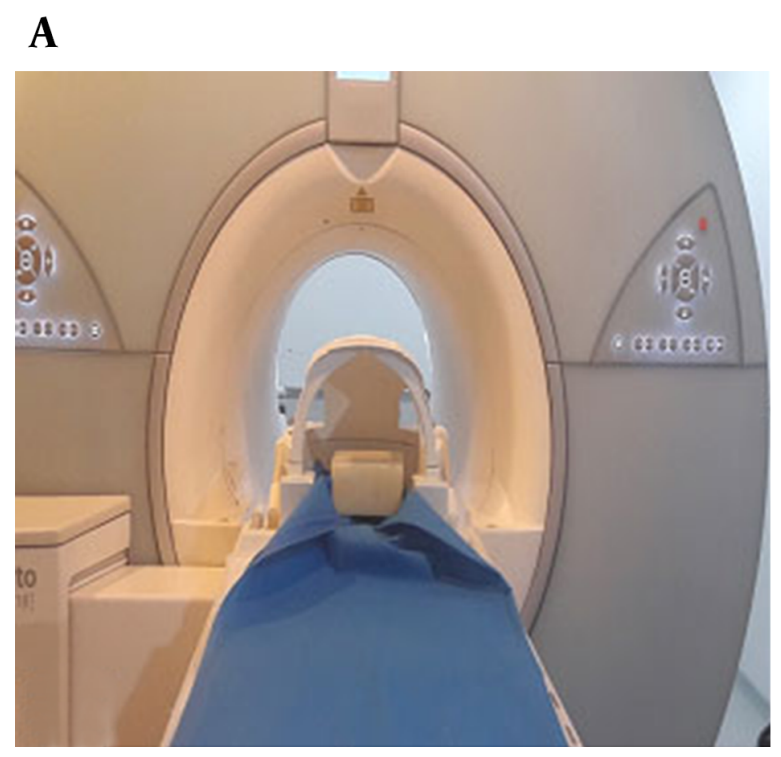

\section{B}
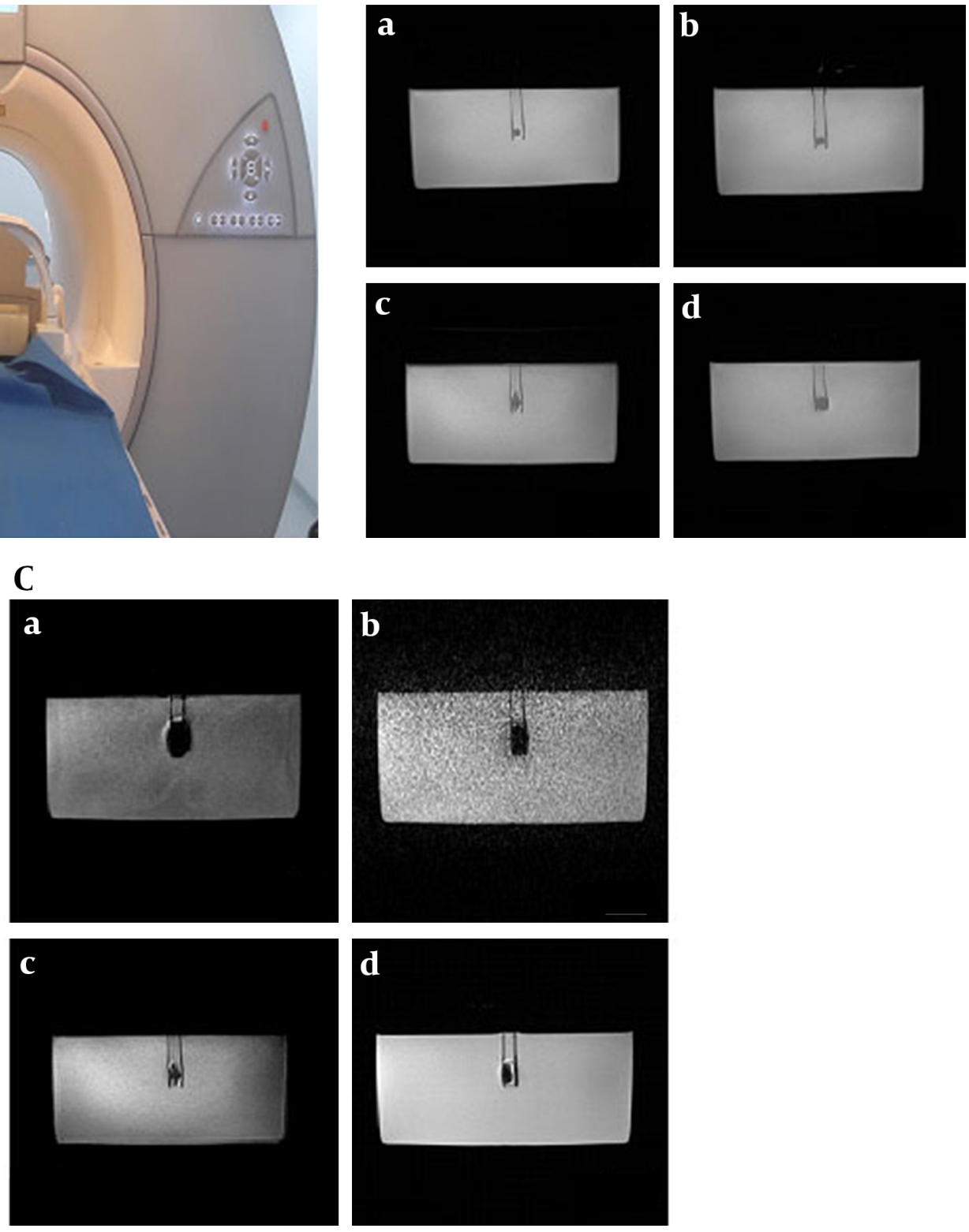

Figure 1. Sample imaging and artifact analysis. A, Phantom filled with paraffin and the dental material placed at the center; B, T1 weighted depiction of ceramic (a), dycal (b), Ti implant (c) and Nickel-chromium (d) materials inside a paraffin phantom acquired by sampling perfection with application optimized contrasts using different flip angle evolution (SPACE) SPACE pulse sequence; C, Artifact representations of the Ti implant acquired by TW Three dimensional fast low-angle shot (3D-FLASH) (a), T2W threedimensional volumetric interpolated breath-hold examination (3D VIBE) (b), T1W 3D SPACE(c) and T1W 2D BLADE(d) pulse sequences.

0.046) such that the volumetric interpolated breath-hold examination (VIBE) sequence resulted in the smallest artifact area.

For dental materials with insignificant artifacts, 2D T2w sequences showed a highly significant difference in artifact size $(\mathrm{P}<0.0001)$ such that the periodically rotated overlapping parallel lines with enhanced reconstruction
(PROPELLER) commonly known as BLADE sequence caused the smallest artifact area possible. A significant difference was also noted in artifact size caused on 3D T2w images of dental materials with insignificant artifact size $(P=0.002)$ such that the SPACE sequence caused the smallest artifact area possible.

The results of ANOVA for Ni-Cr, implant and bracket 
Valizadeh S et al.

Table 1. Characteristics of Parameters Used for Proton Density Weighted, T1-Weighted and T2-Weighted Imaging Sequences

\begin{tabular}{|c|c|c|c|c|c|c|}
\hline \multicolumn{7}{|c|}{ T2-Weighted Images } \\
\hline Description & 2D-TSE & 2D-TrueFISP & 2D-FLAIR & 2D-BLADE & 3D-TSE & 3D-SPACE \\
\hline TR/TE, ms & $2500 / 79$ & $4.28 / 2.14$ & $6000 / 109$ & $2500 / 99$ & $300 / 115$ & $2800 / 381$ \\
\hline TI, ms & - & - & 2019.8 & - & - & - \\
\hline Flip angle & $150 \mathrm{deg}$ & 70 deg & $150 \mathrm{deg}$ & 150 deg & 170 deg & 120 \\
\hline FOV, mm & $206 \times 230$ & $230 \times 230$ & $222 \times 230$ & $260 \times 230$ & $223 \times 223$ & $227 \times 260$ \\
\hline Aq. matrix size & $230 \times 256$ & $256 \times 256$ & $248 \times 256$ & $256 \times 256$ & $256 \times 256$ & $226 \times 256$ \\
\hline Pixel size, mm & $\approx 0.9 \times 0.9 \times 5$ & $\approx 0.9 \times 0.9 \times 5$ & $\approx 0.9 \times 0.9 \times 5$ & $\approx 0.9 \times 0.9 \times 5$ & $\approx 0.9 \times 0.9 \times 1$ & $\approx 1 \times 1 \times 1$ \\
\hline BW, Hz/Px & 190 & 500 & 150 & 360 & 175 & 700 \\
\hline Echo train length & 30 & - & 22 & 35 & 24 & 125 \\
\hline Averages & 1 & 2 & 1 & 1 & 1 & 1 \\
\hline \multicolumn{7}{|c|}{ T1-Weighted Images } \\
\hline Description & 2D-SE & 2D-TSE & 2D-FLASH & \multicolumn{2}{|c|}{ 3D-FLASH } & 3D-VIBE \\
\hline $\mathrm{TR} / \mathrm{TE}, \mathrm{ms}$ & $300 / 8.7$ & $300 / 10$ & $130 / 4.76$ & \multicolumn{2}{|c|}{$24 / 9.56$} & $9.64 / 4.76$ \\
\hline TI, ms & - & - & - & \multicolumn{2}{|c|}{ - } & - \\
\hline Flip angle & $90 \mathrm{deg}$ & $150 \mathrm{deg}$ & 70 deg & \multicolumn{2}{|c|}{$25 \mathrm{deg}$} & $12 \mathrm{deg}$ \\
\hline FOV, $\mathrm{mm}$ & $230 \times 230$ & $230 \times 230$ & $186 \times 230$ & \multicolumn{2}{|c|}{$240 \times 240$} & $230 \times 230$ \\
\hline Aq. matrix size & $512 \times 512$ & $512 \times 512$ & $416 \times 512$ & \multicolumn{2}{|c|}{$512 \times 512$} & $512 \times 512$ \\
\hline Pixel size, mm & $\approx 0.5 \times 0.5 \times 5$ & $\approx 0.5 \times 0.5 \times 5$ & $\approx 0.5 \times 0.5 \times 5$ & \multicolumn{2}{|c|}{$0.5 \times 0.5 \times 1$} & $0.5 \times 0.5 \times 1$ \\
\hline BW, Hz/Px & 150 & 170 & 150 & \multicolumn{2}{|c|}{60} & 200 \\
\hline Echo train length & 1 & 2 & - & \multicolumn{2}{|c|}{ - } & - \\
\hline Averages & 1 & 1 & 1 & \multicolumn{2}{|c|}{1} & 1 \\
\hline \multicolumn{7}{|c|}{ Proton Density Weighted Images } \\
\hline Description & & & \multicolumn{4}{|c|}{ 2D-TSE } \\
\hline TR/TE, ms & & & \multicolumn{4}{|c|}{$2000 / 9.9$} \\
\hline TI, ms & & & \multicolumn{4}{|c|}{ - } \\
\hline Flip angle & & & \multicolumn{4}{|c|}{$150 \mathrm{deg}$} \\
\hline FOV, mm & & & \multicolumn{4}{|c|}{$230 \times 230$} \\
\hline Aq. matrix size & & & \multicolumn{4}{|c|}{$256 \times 256$} \\
\hline Pixel size, mm & & & \multicolumn{4}{|c|}{$0.9 \times 0.9 \times 5$} \\
\hline BW, $\mathbf{H z} / \mathbf{P x}$ & & & \multicolumn{4}{|c|}{190} \\
\hline GRAPPA iPAT & & & \multicolumn{4}{|c|}{2} \\
\hline Echo train length & & & \multicolumn{4}{|c|}{8} \\
\hline Averages & & & \multicolumn{4}{|c|}{1} \\
\hline
\end{tabular}

showed no significant difference $(\mathrm{P}=0.74)$ for artifact size at 2D T1w sequences among these three high artifact materials but TSE sequence caused the lowest size of artifact. Likewise, there was no significant variation $(P=0.598)$ among these materials for artifact at 3D T1w with VIBE sequence possessing the lowest size. On the other hand, a significant variation $(P<0.0001)$ was observed for artifact size produced by these three materials with the lowest ar- 
Table 2. Mean, Standard Deviation, Minimum and Maximum of Artifact Sizes Based on the Studied Materials

\begin{tabular}{|c|c|c|c|c|}
\hline Material & Mean, $\mathbf{m m}^{3}$ & Standard deviation & Minimum & Maximum \\
\hline Ceramic & 189.48 & 153.58 & 51.49 & 689.01 \\
\hline GP Gapadent & 240.76 & 169.43 & 68.73 & 891.75 \\
\hline GP Arident & 268.94 & 147.21 & 75.25 & 580.50 \\
\hline MTA & 231.02 & 155.03 & 63.37 & 664.23 \\
\hline Zinc Phosphate & 246.22 & 145.76 & 68.73 & 633.00 \\
\hline Cavit & 224.37 & 124.62 & .00 & 534.75 \\
\hline Poly Carboxylate & 206.40 & 181.50 & -321.00 & 501.42 \\
\hline Temp Bond & 226.92 & 135.82 & 59.05 & 493.81 \\
\hline Sealer & 210.62 & 98.22 & 87.13 & 393.98 \\
\hline Dycal & 260.66 & 184.14 & 71.63 & 876.54 \\
\hline Composite P4 & 199.89 & 183.34 & 17.42 & 921.00 \\
\hline Composite Z100 & 203.56 & 129.57 & 63.89 & 542.00 \\
\hline Implant TI & 1138.13 & 949.80 & 332.68 & 4416.93 \\
\hline GI Lutting & 366.80 & 544.02 & 123.90 & 3054.03 \\
\hline $\mathrm{Ni}-\mathrm{Cr}$ & 1482.28 & 1397.98 & 220.70 & 6792.66 \\
\hline GI FUGI II & 219.02 & 230.14 & 51.30 & 1232.07 \\
\hline Amalgam & 314.63 & 215.77 & 2.90 & 788.25 \\
\hline Zonalin & 236.85 & 130.32 & 62.92 & 658.98 \\
\hline Bracket & 4307.53 & 4529.93 & 776.26 & 18517.38 \\
\hline
\end{tabular}

tifact belonging to BLADE sequence. Relating to 3D Tw2, Ni$\mathrm{Cr}$, implant and bracket did not differ $(\mathrm{P}=0.993)$ for artifact size, so that either TSE or SPACE sequence can alternatively be used.

In general, in T1w and T2w images of low-artifact dental materials, 2D and 3D sequences can be categorized based on the size of artifacts as follows:

2D - T1w: FLASH $<$ SE $<$ TSE

3D - T1w: VIBE $<$ FLASH

2D - T2w: BALDE $<$ FLAIR $<$ TSE $<<$ TrueFisp

3D - T2W: SPACE $<$ TSE

In T1w and T2w images of high-artifact dental materials, 2D and 3D sequences can be categorized based on the size of artifacts as follows:

2D - T1w: TSE $<$ SE $<$ FLASH

3D - T1w: VIBE $<$ FLASH

2D - T2w: BALDE $<$ FLAIR $<$ TSE $<<$ TrueFisp

3D - T2w: SPACE $\approx$ TSE

The spin echo (SE) pulse sequence is the most frequently employed pulse sequence. As in routine SE imaging, a 90 degree radio frequency pulse is followed by a single 180 degree refocusing pulse. However, in TSE imaging, multiple 180 degree re-focusing pulses are carried out with multiple succeeding SEs. The BLADE is commonly used to reduce the sensitivity to various sources of image artifacts (e.g., motion artifact, field inhomogeneity artifact, eddy current artifact). The SPACE sequence enables acquisition of high resolution 3D datasets with contrasts similar to those acquired from 2D T2-weighted, T1-weighted, proton density and dark fluid protocols at $1.5 \mathrm{~T}$ within a clinically appropriate timeframe. The FLASH is a fast sequence yielding signals known as gradient echo with low flip angles. VIBE sequence is a modified form of FLASH sequence, which allows dynamic and high-resolution imaging at 30 seconds breath-hold. The true fast imaging with steady state precession (true FISP) aids the technique to become reliable through speed and relative motion insensitivity acquisition, even in patients who have difficulty with holding their breath.

Different types of imaging sequences including inversion recovery, spin echo and gradient echo were statically compared and the results revealed significant differences among them (data not shown). These sequences were the routine sequences, applicable in maxillofacial MR imaging. The inversion recovery sequences included fluid attenuation inversion recovery (FLAIR). The spin echo sequences included turbo spin echo, 3D turbo spin echo, BLADE and 3D space. Gradient echo sequences included 2D FLASH, 


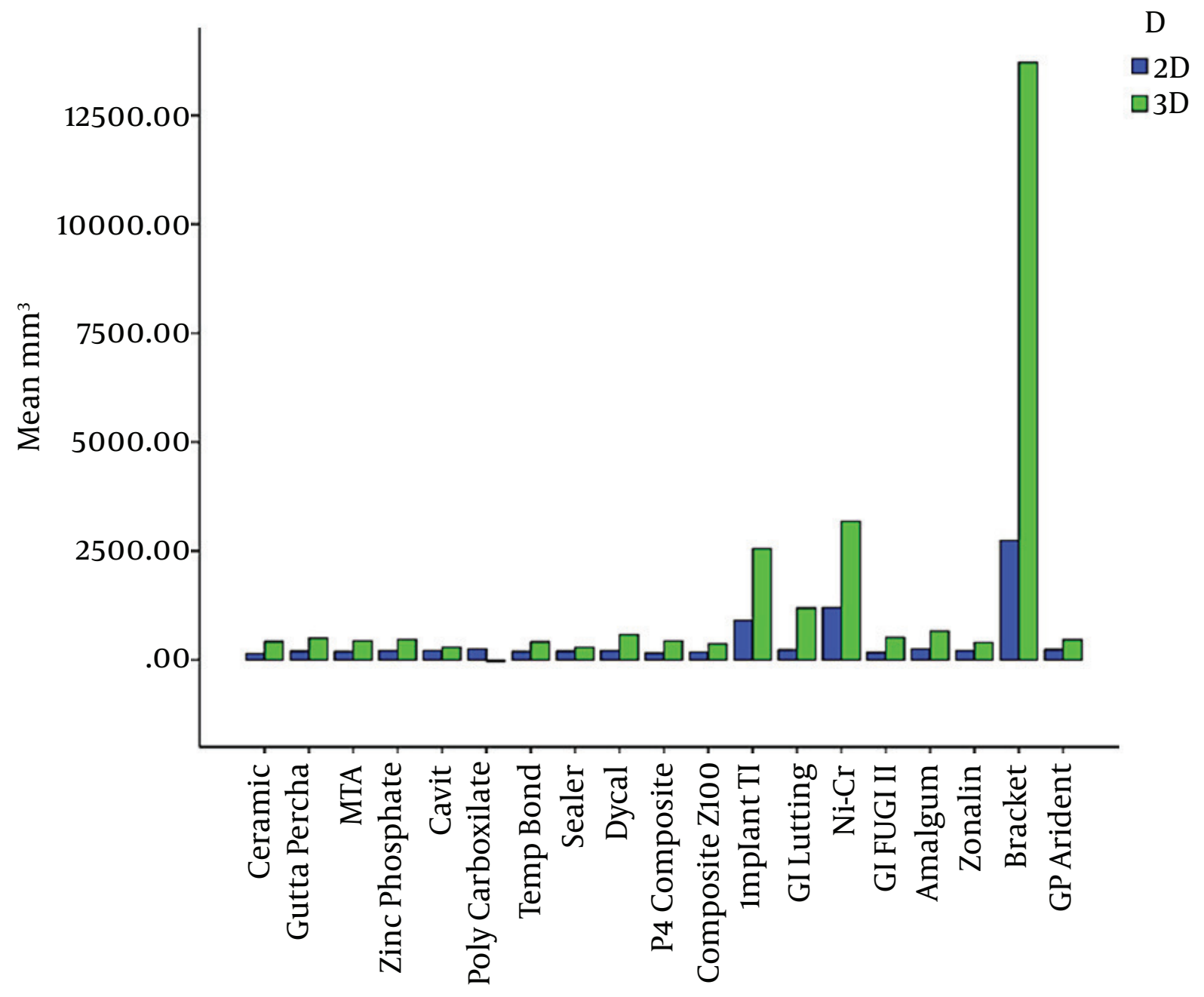

Material

Figure 2. Comparison of the mean size of blooming artifacts on two dimensional (2D) and three dimensional (3D-images based on the type of dental material

true FISP, 3D FLASH and 3D VIBE. Evaluation of the size of artifacts in different imaging sequences revealed that inversion recovery caused the smallest and gradient echo caused the largest artifact areas. Pairwise comparison of different imaging sequences by Tukey's post-hoc test revealed significant differences between spin echo and gradient echo $(P=0.0006)$ and also between inversion recovery and gradient echo $(\mathrm{P}=0.0056)$ in terms of size of artifacts.

\section{Discussion}

In the current in vitro analysis, we evaluated 19 maxillofacial implantable materials and found that the bracket, titanium implants, and the Ni-Cr materials are the three most susceptible materials to external Bo magnetic field; thus creating huge metal-induced artifacts. The Siemens default 2D-TSE, 2D-BLADE, 3D-VIBE, and 3D-SPACE are the most appropriate pulse sequences for the 2D-T1w, 2D-T2w, 3D-T1w, and 3D-T2w evaluations of the bracket/titanium/Ni$\mathrm{Cr}$ containing structures, respectively. High flip angles/echo train lengths/receiver bandwidths/in-plane resolution/ through-plane resolution can reduce the metallic objects induced artifacts. Likewise, the k-space filling trajectory and the preparation RF pulses can reduce the susceptibility artifacts sizes largely.

Presence of ferromagnetic metals in some dental materials causes magnetic field inhomogeneity in MRI. Metal substances cause a magnetic field and significantly change 


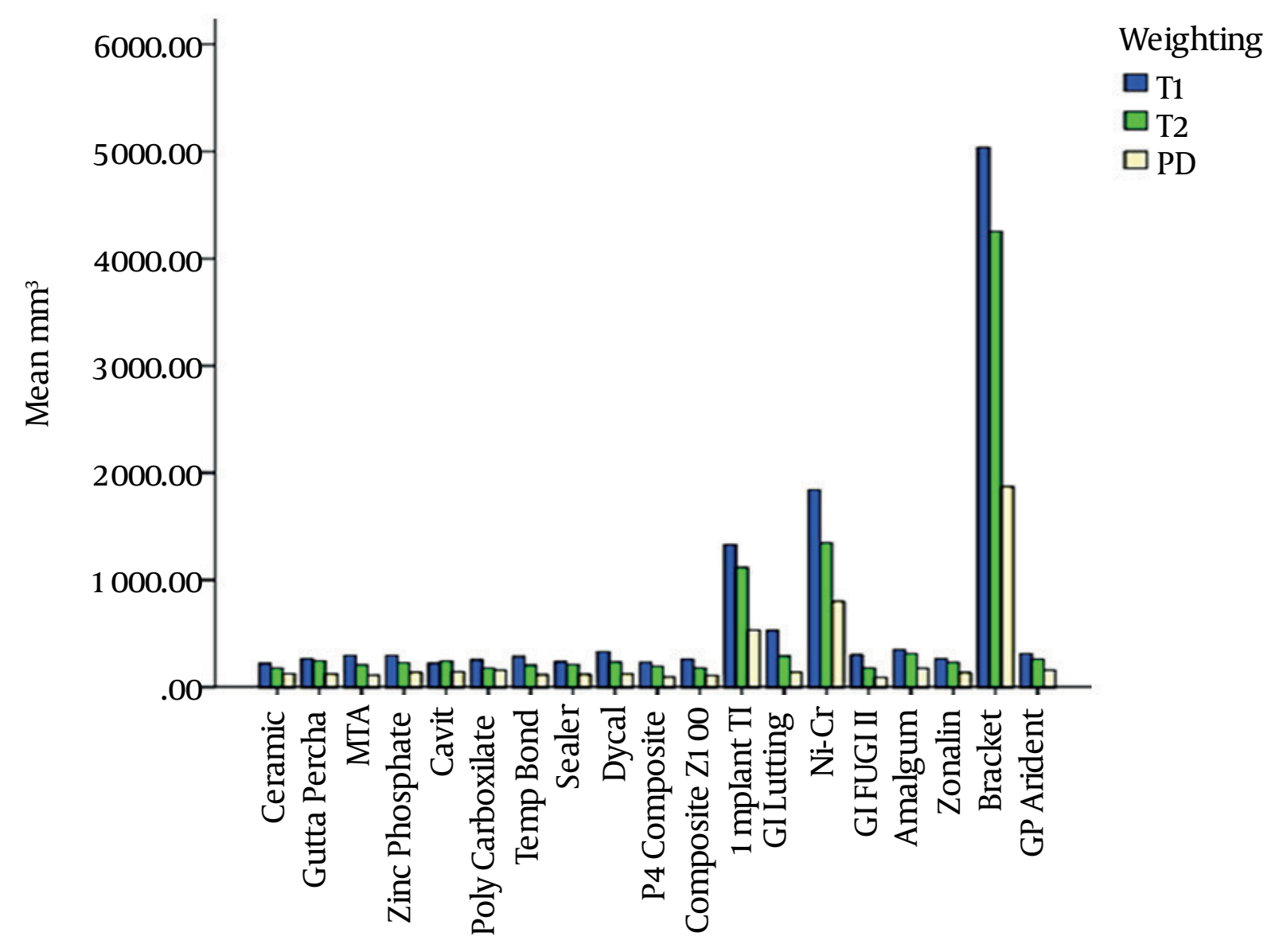

\section{Matenial}

the precession frequencies of protons present in the adjacent tissue. Tissues adjacent to ferromagnetic compounds are affected by the metal magnetic field and resultantly, precession does not occur in them or occurs with a different frequency and thus, a useful signal is not produced (5). Nonetheless, the current study showed that in addition to metallic dental materials, non-metallic materials also created artifacts, which is due to the presence of ferromagnetic metal components in their composition. The current results were in agreement with those of Eggers et al. (7) who reported that minute amounts of ferromagnetic materials can significantly attenuate signal void.

In the current study, all tested dental materials created artifacts; among which, ceramic and orthodontic metal brackets created the smallest and largest artifact areas, respectively. Significant artifacts created by orthodontic metal brackets have also been reported in previous stud- ies (6, 8-13). Insignificant artifacts caused by ceramics were also reported by Asano et al. (8) and Beau et al. (9) who stated that ceramic brackets should not be necessarily removed from the oral cavity prior to MRI. Cox et al. (11) showed that metal-free ceramic orthodontic appliances do not cause any distortion on MR images with ultra-short echo time.

In our study, the size of amalgam artifacts was small, which was in line with the findings of previous studies in this respect $(6,14,15)$. However, somewhat contradictory findings have been published concerning the intensity of image artifacts produced by different dental materials ( 7 , 10, 14-16). For instance, inconsistent findings have been stated for amalgam, titanium, and other dental materials (6). Nonetheless, there are many factors involved in the severity of artifacts including echo time, pulse sequence, magnetic field strength, imaging plane, volume and shape 
Table 3. Optimal Conditions and Parameters of Imaging to Minimize Artifacts Caused by Each Type of Dental Material

\begin{tabular}{|c|c|c|c|c|}
\hline Material & 2D-T1 & 2D-T2 & 3D-T1 & 3D-T2 \\
\hline Ceramic & SE & BLADE & VIBE & SPACE \\
\hline Gutta percha Gapadent & SE/FLASH & BLADE & VIBE & SPACE \\
\hline Gutta percha Arident & FLASH & BLADE & VIBE & SPACE \\
\hline MTA & SE/FLASH & BLADE & VIBE & SPACE \\
\hline Zinc-Phosphate & FLASH & TSE & VIBE & SPACE \\
\hline Cavit & FLASH & FLAIR & FLASH & SPACE \\
\hline Poly carboxylate & FLASH & TSE/BLADE & FLASH & SPACE \\
\hline Temp Band & FLASH & FLAIR & FLASH/VIBE & SPACE \\
\hline Sealer & FLASH & BLADE & VIBE & SPACE/TSE \\
\hline Dycal & FLASH & FLAIR/BLADE & VIBE & SPACE \\
\hline Composite P4 & FLASH & FLAIR/TSE/BLADE & VIBE & SPACE \\
\hline Composite Z100 & FLASH & FLAIR & VIBE & SPACE \\
\hline TI Implant & TSE & TSE & VIBE & SPACE \\
\hline GI Chemfil & FLASH & BLADE & VIBE & SPACE \\
\hline GI Fuji II & FLASH & FLAIR & VIBE & SPACE \\
\hline Amalgam & FLASH & BLADE & VIBE & SPACE \\
\hline Zonalin & FLASH & FLAIR/BLADE & VIBE & TSE \\
\hline Bracket & TSE & BLADE & VIBE & TSE \\
\hline $\mathrm{Ni}-\mathrm{Cr}$ & TSE & BLADE & VIBE & SPACE/TSE \\
\hline
\end{tabular}

Abbreviations: BW, Bandwidth; 2D-FLAIR, Two dimensional fluid attenuated inversion recovery; 2D-TSE, Two dimensional -turbo spin echo; 3D-SPACE; Three dimensional -sampling perfection with application optimized contrasts using different flip-angle evolution; iPAT, integrated parallel acquisition techniques; SE, Spin echo; TE, Echo time; TI, Inversion time; TR, Repetition time; true FISP, true Fast imaging with steady-state precession; FOV, Field of view; VIBE, Volumetric interpolated breath-hold examination.

of the material, image resolution and the associated gradient field strength, and also the distance between the material and the desired object (6).

The spatial resolution would improve if we could carry out this experiment under in vivo conditions. However, constructing such an experiment under in vivo conditions would be difficult due to the prolonged scanning time and obvious difficulty with obtaining human subjects. In addition, we have to address the other limitations of our study, which were inaccessibility to other MRI devices as well as employing different compatible defaults to those devices.

In the current study, the size of artifact in gradient recalled echo images was significantly larger than that on spin-echo images, which was in accordance with previous findings $(5,15-21)$ This finding may be due to the fact that spin-echo sequences have a 180-degree pulse that refocuses the frequency shifts due to susceptibility in echo time (TE) and thus, eliminates pixel intensity shifts (22). Moreover, in gradient echo (GE) sequence, magnetic field slightly superimposes on the main field and thus, it is the most sensitive sequence to metals (23).

\subsection{In Conclusion}

In presence of highly susceptible materials in oral cavity, 3D SPACE sequence is recommended for use in MR sialography. In addition, when using heavily T1-weighted gadolinium-enhanced MR imaging, T1 VIBE is the most suitable sequence. Further study is required to test the MR imaging suitability of the same functioning maxillofacial implantable materials in the field of maxillofacial radiology, using same functioning implantable instruments and MRI devices.

\section{Supplementary Material}

Supplementary material(s) is available here [To read supplementary materials, please refer to the journal website and open PDF/HTML].

\section{Footnotes}

Authors' Contributions: None declared. 
Financial Disclosure: None declared.

Funding/Support: None declared.

\section{References}

1. Korn P, Elschner C, Schulz MC, Range U, Mai R, Scheler U. MRI and dental implantology: two which do not exclude each other. Biomaterials. 2015;53:634-45. doi: 10.1016/j.biomaterials.2015.02.114. [PubMed: 25890759].

2. Geibel MA, Schreiber ES, Bracher AK, Hell E, Ulrici J, Sailer LK, et al. Assessment of apical periodontitis by MRI: a feasibility study. Rofo. 2015;187(4):269-75. doi: 10.1055/s-0034-1385808. [PubMed: 25594373].

3. Talbot BS, Weinberg EP. MR Imaging with Metal-suppression Sequences for Evaluation of Total Joint Arthroplasty. Radiographics. 2016;36(1):209-25. doi: 10.1148/rg.2016150075. [PubMed: 26587889].

4. Ladefoged CN, Andersen FL, Keller SH, Beyer T, Law I, Hojgaard L, et al. Automatic correction of dental artifacts in PET/MRI.J Med Imaging (Bellingham). 2015;2(2):24009. doi: 10.1117/1.JMI.2.2.024009. [PubMed: 26158104].

5. Klinke T, Daboul A, Maron J, Gredes T, Puls R, Jaghsi A, et al. Artifacts in magnetic resonance imaging and computed tomography caused by dental materials. PLoS One. 2012;7(2). e31766. doi: 10.1371/journal.pone.0031766. [PubMed: 22384071].

6. Tymofiyeva O, Vaegler S, Rottner K, Boldt J, Hopfgartner AJ, Proff PC, et al. Influence of dental materials on dental MRI. Dentomaxillofac Radiol. 2013;42(6):20120271. doi: 10.1259/dmfr.20120271. [PubMed: 23610088].

7. Eggers G, Rieker M, Kress B, Fiebach J, Dickhaus H, Hassfeld S. Artefacts in magnetic resonance imaging caused by dental material. MAGMA. 2005;18(2):103-11. doi: 10.1007/s10334-005-0101-0. [PubMed: 15785943].

8. Asano S, Kaneda T, Fukuda T, Makiyama Y, Hirota H, Saitoh K, et al. Influence of metal artifact by orthodontic appliances on brain MRI. Int J Oral Med Sci. 2016;14(4):74-81. doi: 10.5466/ijoms.14.74.

9. Beau A, Bossard D, Gebeile-Chauty S. Magnetic resonance imaging artefacts and fixed orthodontic attachments. Eur J Orthod. 2015;37(1):105-10. doi:10.1093/ejo/cju020. [PubMed: 24997025].

10. Costa AL, Appenzeller S, Yasuda CL, Pereira FR, Zanardi VA, Cendes F. Artifacts in brain magnetic resonance imaging due to metallic dental objects. Med Oral Patol Oral Cir Bucal.2009;14(6):E278-82. [PubMed: 19300375].

11. Cox RJ, Kau CH, Rasche V. Three-dimensional ultrashort echo magnetic resonance imaging of orthodontic appliances in the natural dentition. Am J Orthod Dentofacial Orthop. 2012;142(4):552-61. doi: 10.1016/j.ajodo.2012.03.033. [PubMed: 22999679].
12. Elison JM, Leggitt VL, Thomson M, Oyoyo U, Wycliffe ND. Influence of common orthodontic appliances on the diagnostic quality of cranial magnetic resonance images. Am J Orthod Dentofacial Orthop. 2008;134(4):563-72. doi: 10.1016/j.ajodo.2006.10.038. [PubMed: 18929275].

13. Okano Y, Yamashiro M, Kaneda T, Kasai K. Magnetic resonance imaging diagnosis of the temporomandibular joint in patients with orthodontic appliances. Oral Surg Oral Med Oral Pathol Oral Radiol Endod. 2003;95(2):255-63. doi: 10.1067/moe.2003.37. [PubMed: 12582369].

14. Abbaszadeh K, Heffez LB, Mafee MF. Effect of interference of metallic objects on interpretation of T1-weighted magnetic resonance images in the maxillofacial region. Oral Surg Oral Med Oral Pathol Oral Radio Endod. 2000;89(6):759-65. [PubMed: 10846134].

15. Starcukova J, Starcuk ZJ, Hubalkova H, Linetskiy I. Magnetic susceptibility and electrical conductivity of metallic dental materials and their impact on MR imaging artifacts. Dent Mater. 2008;24(6):715-23. doi:10.1016/j.dental.2007.07.002. [PubMed: 17884157].

16. Shafiei F, Honda E, Takahashi H, Sasaki T. Artifacts from dental casting alloys in magnetic resonance imaging. I Dent Res. 2003;82(8):602-6. doi: 10.1177/154405910308200806. [PubMed: 12885843].

17. Chen DP, Wu GY, Wang YN. Influence of galvano-ceramic and metalceramic crowns on magnetic resonance imaging. Chin Med J (Engl). 2010;123(2):208-11. [PubMed: 20137372].

18. Cortes AR, Abdala-Junior R, Weber M, Arita ES, Ackerman JL. Influence of pulse sequence parameters at $1.5 \mathrm{~T}$ and 3.0 T on MRI artefacts produced by metal-ceramic restorations. Dentomaxillofac Radiol. 2015;44(8):20150136. doi: 10.1259/dmfr.20150136. [PubMed: 26084475].

19. Imai H, Tanaka Y, Nomura N, Tsutsumi Y, Doi H, Kanno Z, et al. Threedimensional quantification of susceptibility artifacts from various metals in magnetic resonance images. Acta Biomater. 2013;9(9):84339. doi: 10.1016/j.actbio.2013.05.017. [PubMed: 23707948].

20. Murakami S, Verdonschot RG, Kataoka M, Kakimoto N, Shimamoto $\mathrm{H}$, Kreiborg S. A standardized evaluation of artefacts from metallic compounds during fast MR imaging. Dentomaxillofac Radiol 2016;45(8):20160094. doi: 10.1259/dmfr.20160094. [PubMed: 27459058].

21. Stradiotti P, Curti A, Castellazzi G, Zerbi A. Metal-related artifacts in instrumented spine. Techniques for reducing artifacts in CT and MRI: state of the art. Eur Spine J. 2009;18 Suppl 1:102-8. doi: 10.1007/s00586009-0998-5. [PubMed: 19437043]

22. Lee MJ, Kim S, Lee SA, Song HT, Huh YM, Kim DH, et al. Overcoming artifacts from metallic orthopedic implants at high-field-strength MR imaging and multi-detector CT. Radiographics. 2007;27(3):791-803. doi: 10.1148/rg.273065087. [PubMed: 17495293].

23. Hashemi RH, Bradley WG, Lisanti CJ. MRI: The Basics. 2nd ed.1. Philadelphia: Lippincott Williams \& Wilkins; 2004 